\title{
Combinatorial and sequential delivery of gemcitabine and oseltamivir phosphate from implantable poly(D,L-lactic-co-glycolic acid) cylinders disables human pancreatic cancer cell survival
}

This article was published in the following Dove Press journal:

Drug Design, Development and Therapy

31 July 2017

Number of times this article has been viewed

\author{
Stephanie Allison Logan' \\ Amanda J Brissenden' \\ Myron R Szewczuk ${ }^{2}$ \\ Ronald J Neufeld' \\ 'Department of Chemical Engineering, \\ ${ }^{2}$ Department of Biomedical and \\ Molecular Sciences, Queen's \\ University, Kingston, ON, Canada
}

Correspondence: Ronald J Neufeld Department of Chemical Engineering, Queen's University, 19 Division Street, Bioscience Complex, Room I443A, Kingston, ON K7L 3N6, Canada

$\mathrm{Tel}+\mathrm{I} 6135532827$

Fax +l 6135336637

Email neufeld@queensu.ca

Myron R Szewczuk Department of Biomedical and Molecular Sciences, Queen's University, 18 Stuart Street, Botterell Hall, Room 827,

Kingston, ON K7L 3N6, Canada

Tel +l 6135332457

Fax +l 6135336796

Email szewczuk@queensu.ca
Abstract: Combination therapies against multiple targets are currently being developed to prevent resistance to a single chemotherapeutic agent and to extirpate pre-existing resistance in heterogeneous cancer cells in tumors due to selective pressure from the single agent. Gemcitabine (GEM), a chemotherapeutic agent, is the current standard of care for patients with pancreatic cancer. Patients with pancreatic cancer receiving GEM have a low progression-free survival. Given the poor response rate to GEM, cancer cells are known to develop rapid resistance to this drug. Metronomic chemotherapy using combinatorial and sequential delivery systems are novel developmental approaches to disrupt tumor neovascularization, reduce systemic drug toxicity, and increase the sensitivity of chemotherapeutics in cancer. Here, implantable double-layered poly(D,L-lactic-co-glycolic acid) (PLGA) cylinders were engineered to sequentially release GEM in combination with oseltamivir phosphate (OP) over an extended time. Double-layered PLGA cylindrical implants loaded with these active hydrophilic drugs were fabricated with minimal loss of drugs during the formulation, enabling extensive control of drug loading and establishing uniform drug distribution throughout the polymer matrix. OP is used in the formulation because of its anticancer drug properties targeting mammalian neuraminidase 1 (Neu1) involved in multistage tumorigenesis. OP and GEM encapsulated in inner/outer $\mathrm{GEM}^{\text {in }} / \mathrm{OP}^{\text {out }}$ or $\mathrm{OP}^{\text {in }} / \mathrm{GEM}^{\text {out }}$ implantable double-layered PLGA cylinders displayed sustained near linear release over 30 days. OP and GEM released from the double-layered PLGA cylinders effectively reduced cell viability in pancreatic cancer cell line PANC1 and its GEM-resistant variant for up to 15 days.

Keywords: pancreatic cancer, oseltamivir phosphate, gemcitabine, PLGA, chemoresistance

\section{Introduction}

Implantable chemotherapeutic delivery systems are designed to provide sustained release of a drug at the tumor site, providing an optimal dosing for a continuous therapeutic effect while reducing adverse effects associated with systemic chemotherapy. ${ }^{1}$ Metronomic chemotherapy, which involves lower, more frequent dosing of drug, has resulted in reduced tumor volumes and fewer adverse effects than standard chemotherapy in murine models of pancreatic cancer. ${ }^{2-4}$ Furthermore, in patients with pancreatic ductal adenocarcinoma (PDAC), there is an elevated frequency of acquired chemoresistance, which has been linked to highly penetrant genetic mutations at various loci, including Kirsten ras (KRAS) oncogene, tumor suppressor p53, cyclin-dependent 
kinase inhibitor 2A (CDKN2A), and mothers against decapentaplegic homolog-4/deleted in pancreatic cancer-4 (smad4/ DPC4). ${ }^{5}$ Gemcitabine (GEM) hydrochloride (20,20-difluoro20-deoxycytidine or $\mathrm{dFdC}$ ) is a hydrophilic chemotherapeutic drug, which is used as the standard of care for patients with unresectable pancreatic cancer. However, cancer resistance to GEM is a major problem during patient treatment. ${ }^{6}$ Recently, we reported that oseltamivir phosphate (OP) is an effective anticancer agent capable of sensitizing GEM-resistant pancreatic cancer cells to GEM, thereby increasing the efficacy of the chemotherapeutic agent. ${ }^{7,8}$ In addition, we have reported that poly(D,L-lactic-co-glycolic acid) (PLGA)-loaded OP cylinders surgically implanted at the tumor site in a $\mathrm{RAG} 2 \mathrm{xC} \gamma$ double mutant mouse model of human pancreatic cancer inhibited not only tumor growth but also tumor neovascularization and metastasis to the liver and lungs compared with the untreated cohort over the 30 days release period. ${ }^{9}$ The xenograft human pancreatic tumors from PLGA-OPtreated cohorts also expressed significantly higher levels of E-cadherin with concomitant reduced N-cadherin and host $\mathrm{CD} 31^{+}$endothelial cells compared to the untreated cohort. We also reported sustained release of OP over 30 days from the implantable PLGA-OP cylinder. ${ }^{9}$ Despite difficulties with the encapsulation of small hydrophilic drugs, particulate OP encapsulation within polymeric PLGA cylinders using the formulation method described in these studies resulted in full retention of the drug. These results clearly indicated that OP delivered from PLGA cylinders surgically implanted at the tumor site shows promise as an effective treatment therapy for pancreatic cancer. To this end, we defined the optimal combinations and/or sequences of GEM with the novel OP therapy, which may be a more effective treatment regimen than with GEM alone in preventing acquired chemoresistance.

One of the important challenges in drug delivery is the difficulty inherent in the full encapsulation and retention, followed by long-term and targeted delivery of small molecular weight, hydrophilic therapeutics at a tumor site. PLGA is a copolymer composed of lactic and glycolic acid monomers and has been used as a drug delivery vehicle. PLGA is susceptible to hydrolytic degradation of the ester linkage on the polymer backbone, which results in release of the encapsulated drug. ${ }^{10}$ PLGA has been used to encapsulate a wide range of therapeutics, and several PLGA drug delivery applications, such as Lupron Depot, Risperdal Conta, and Zoladex, have been approved by the US Food and Drug Administration, European Medicine Agency, and Health Canada. ${ }^{11}$ However, therapeutics demonstrating extended term, sustained release (weeks or longer) from PLGA polymer are hydrophobic in nature with molecular weights of at least $400 \mathrm{~g} / \mathrm{mol}$, with most being $>1,000 \mathrm{~g} / \mathrm{mol}$. Extended term, sustained release of small molecular weight hydrophilic chemotherapeutics from PLGA is thus a challenge to achieve.

In the present study, double-layered PLGA GEM and OP-loaded cylindrical implants were designed and engineered to provide an optimal combinatorial and sequential sustained release of the hydrophilic drugs for 30 days. This report describes the efficacy of this unique delivery system in the treatment of pancreatic cancer cells and its ability to disable the survival mechanism of pancreatic cancer with acquired chemoresistance. Here, OP and GEM encapsulated in distinct inner/outer layers of implantable double-layered PLGA cylinders disabled pancreatic cancer cell survival and increased sensitivity to therapy involving combinatorial and sequential delivery of two small molecular weight hydrophilic chemotherapeutics.

\section{Materials and methods \\ Reagents}

GEM hydrochloride was obtained from Sigma-Aldrich Co. (St Louis, MO, USA). OP with $98 \%$ purity was obtained from Hangzhou DayangChem Co., Ltd (Hangzhou, China). PLGA (50/50) was obtained from Purac Biomaterials (Gorinchem, the Netherlands). The molecular weight was determined by gel permeation chromatography (Viscotek GPCmas VE 2001; Malvern Instruments, Malvern, UK) and calibrated with poly(ethylene oxide) standards. PLGA was determined to have a molecular weight $\left(M_{\mathrm{W}}\right)$ of 16,400 $\left(M_{\mathrm{W}} / M_{\mathrm{n}}=1.58\right)$.

\section{Cell lines}

PANC1 (human epithelioid carcinoma, epithelial-like, ATCC $^{\circledR}$ CRL-1469'TM) was purchased from the American Type Culture Collection (ATCC) (Manassas, VA, USA). Cells were grown at $37^{\circ} \mathrm{C}$ with $5 \% \mathrm{CO}_{2}$ and cultured with $1 \times$ Dulbecco's Modified Eagle's Medium (DMEM) containing $10 \%$ fetal bovine serum (FBS) and $5 \mu \mathrm{g} / \mathrm{mL}$ Plasmocin ${ }^{\mathrm{TM}}$. PANC1 cells resistant to GEM (PANC1-GEMR) were developed by culturing PANC1 cells in $1 \times$ DMEM containing $10 \%$ FBS and $5 \mu \mathrm{g} / \mathrm{mL}$ Plasmocin and gradually increasing the concentrations of GEM up to $0.01 \mu \mathrm{M}$. PANC1-GEMR cells are stable and have been cultured in conditioned medium containing $0.01 \mu \mathrm{M}$ GEM for $>3$ years.

\section{OP and GEM detection}

A 1260 Infinity high performance liquid chromatography (HPLC) and a Poroshell 120 SB-C18 threaded column (Agilent 
Technologies, Santa Clara, CA, USA) 4.6×50 mm (2.7 $\mu \mathrm{m})$ were used. OP (Hangzhou Dayang Chem Co., Ltd) and GEM were dissolved in HPLC grade methanol. The mobile phase was $60 \%$ HPLC grade methanol and $40 \% 0.04 \mathrm{M}$ ammonium acetate buffer (pH 5.2) at a flow rate of $1 \mathrm{~mL} / \mathrm{min}$. The column temperature was $25^{\circ} \mathrm{C}$, the injection volume was $20 \mu \mathrm{L}$, and $\mathrm{OP}$ and GEM were detected at $230 \mathrm{~nm}$.

\section{Formulation of cylinders containing OP and GEM}

PLGA (80 mg), 4-6 mg sorbitan monooleate (Span 80), and the desired drug were mixed together in $400 \mu \mathrm{L}$ acetone. The solution was ejected dropwise onto a Teflon sheet and then stored at $20^{\circ} \mathrm{C}$ for $72 \mathrm{~h}$. The polymer film was removed from the Teflon sheet using a razor blade and rolled around a $16 \mathrm{G}$ needle tip. The razor blade and needle tip were lubricated with glycerol to prevent the film from sticking. Once rolled, the hollow polymer cylinder was removed from the needle tip. To form a double cylinder, a second layer was rolled around the first cylinder. OP and GEM double-layered PLGA cylinders were produced and compared with blank control cylinders that did not contain drug.

\section{Scanning electron microscopy (SEM)}

Samples were fixed to aluminum inserts with carbon tape and gold sputtered. SEM was performed using a JEOL 840 (Tokyo, Japan) with an accelerating voltage of $10 \mathrm{kV}$.

\section{Release kinetic experiments}

PLGA cylinders were suspended in $5 \mathrm{~mL}$ of $0.1 \mathrm{M}$ sodium phosphate buffer $(\mathrm{pH} 7.4)$ and stored at $37^{\circ} \mathrm{C}$. Supernatants were extracted periodically and replaced with fresh buffer. Supernatants were stored at $-20^{\circ} \mathrm{C}$ pending analysis. OP and GEM released from cylinders were reported as a percent of the cumulative drug released after 30 days.

\section{PLGA cylinders in cell culture}

PANC1 and PANC1-GEMR cells were plated in $25 \mathrm{~cm}^{2}$ cell culture flasks at $\sim 112,500$ and 45,000 cells per flask, respectively, and incubated overnight. PANC1-GEMR cells were plated at a lower density due to a faster growth rate. Experiments were carried out for 3, 6, 10, or 15 days. Experimental cohorts contained a control (no drug) or drugloaded double-layered PLGA cylinder and untreated cells not exposed to a cylinder. Drug-loaded cylinders contained $3 \mathrm{mg}$ of GEM and $16 \mathrm{mg}$ of OP. Medium was changed every 3 days. On the final day, cells were lifted using TrypLE Express (Thermo Fisher Scientific, Waltham, MA, USA) and counted twice using a hemocytometer. Trypan blue stain was added to cells prior to counting to ensure that only viable cells were counted. Cell viability was measured as cell count, percentage of the control.

\section{Statistical analysis}

Graphing and statistical analysis were carried out using GraphPad Prism 5. Statistical analyses of the data used unpaired $t$-tests at $95 \%$ confidence.

\section{Results and discussion Fabrication and characterization of double-layered PLGA cylinders loaded with OP and GEM}

Double-layered PLGA cylinders loaded with OP and GEM at different doses were fabricated using a previous reported method as depicted in Figure 1 as described for singlelayered cylinders by Hrynyk et al. ${ }^{9}$ Implantable doublelayered PLGA cylinders were produced first by dissolving PLGA in acetone. OP and GEM are insoluble in acetone and, thus, were dispersed into the PLGA solution as insoluble particulates facilitated by addition of Span 80 . The suspension was then ejected dropwise onto a Teflon sheet, forming a thin film enabling evaporation of the acetone. After drying, films were formed into single- or double-layered PLGA cylinders around a $16 \mathrm{G}$ needle. Cylinders were loaded with $16 \mathrm{mg}$ of OP or $3 \mathrm{mg}$ of GEM, the dosage of which is based on prior preclinical in vivo animal studies. Granular OP and GEM were distributed throughout the polymer matrix (Figure 2). The double-layered PLGA cylinders measured $\sim 10 \times 5 \mathrm{~mm}$ (Figure 2A). A cross-section of a control cylinder without drug is shown in Figure 2B, which illustrates the distinct polymer layers resulting from the formulation method. The control empty cylinder appears smooth in Figure 2B. SEM micrographs of cross-section, surface, and internal morphologies of PLGA-OP/GEM cylinders show evenly dispersed particles of pure OP of $\sim 1-5 \mu \mathrm{m}$ visibly embedded in the polymer film in Figure 2C. Larger GEM particles are visible embedded in the polymer film in Figure 2D. The cylinder appears smooth and continuous as seen in Figure 2A.

The degradable double-layered PLGA implants were designed to release OP and GEM for a period extending up to 30 days. Release profiles of OP and GEM from PLGA were determined, and the stability of the respective drugs in terms of the sustained ability to elicit a cancer cell response was also determined. It is noteworthy that both OP and GEM have not been investigated for long-term stability due to their usual route of administration. In particular, OP is 


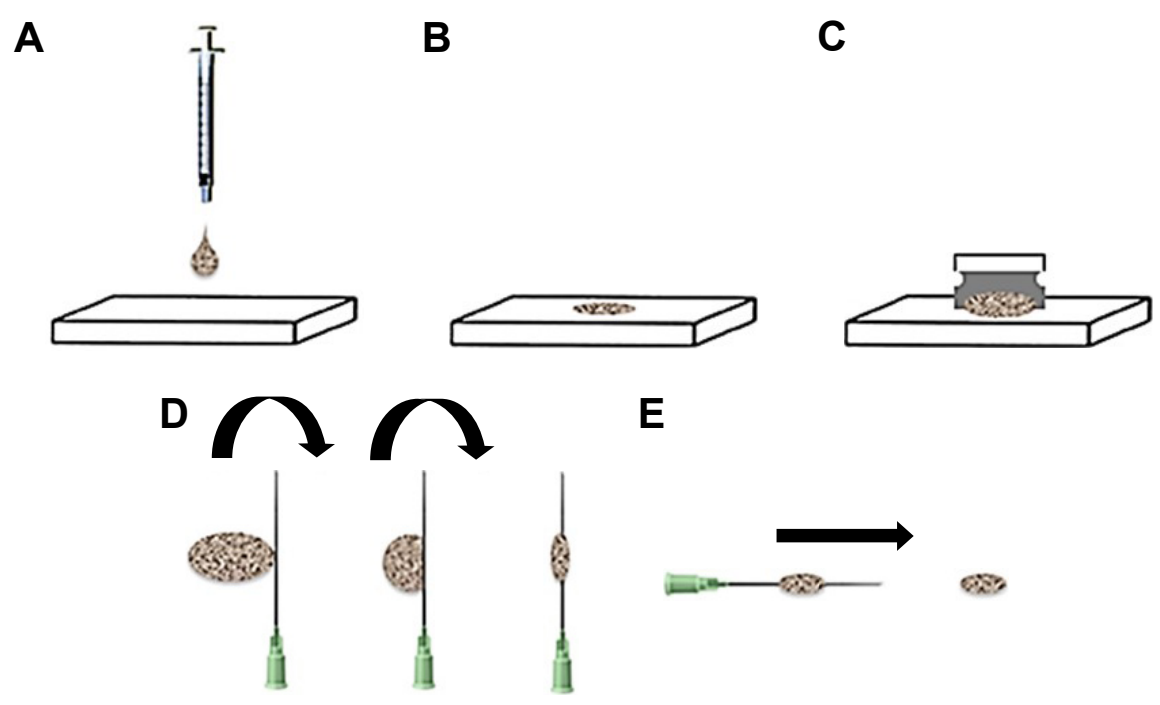

Figure I Therapeutic design of OP- and GEM-loaded double-layered PLGA cylindrical implants.

Notes: Production of a double-layered PLGA. (A) Insoluble drug particles were suspended in the PLGA, Span 80, and acetone solution and ejected dropwise onto a Teflon sheet. (B) Acetone evaporates from the suspension, resulting in a thin, malleable film. (C) The film is scraped off the Teflon sheet using a razor blade and (D) wrapped around a glycerol-lubricated $16 \mathrm{G}$ needle to form a cylinder. (E) The cylinder is then removed from the needle. A double-layered PLGA cylinder is formed by rolling a second film around the first cylinder. Adapted from Hrynyk M, Ellis JP, Haxho F, et al. Therapeutic designed poly (lactic-co-glycolic acid) cylindrical oseltamivir phosphate-loaded implants impede tumor neovascularization, growth and metastasis in mouse model of human pancreatic carcinoma. Drug Des Devel Ther. 20I5;9:4573-4586. ${ }^{9}$

Abbreviations: GEM, gemcitabine; OP, oseltamivir phosphate; PLGA, poly(D,L-lactic-co-glycolic acid).

administered orally as a tablet while GEM is administered intravenously over a period of $\sim 30 \mathrm{~min}$. To this end, it was necessary to determine the stability of these hydrophilic drugs over an extended release period before encapsulation in double-layered PLGA cylinders. GEM and OP with chemical structures depicted in Figure 3 were assayed for their stability in phosphate buffer ( $\mathrm{pH} 7.4$ ) at $37^{\circ} \mathrm{C}$ for 30 days using HPLC analysis.

No significant change in the concentration of GEM was detected by HPLC over 30 days. However, a slight $30 \%$
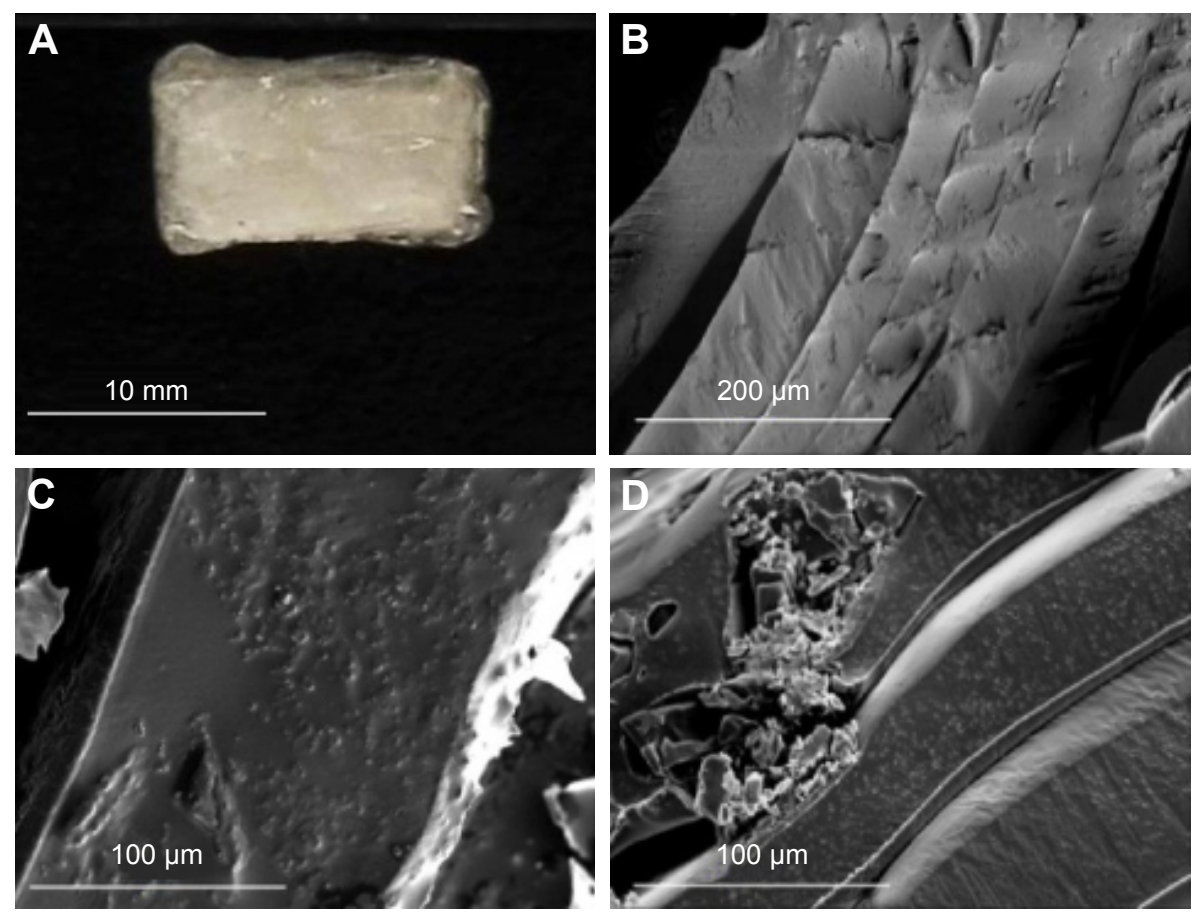

Figure 2 Double-layered PLGA cylinder with 16 mg OP inner layer and 3 mg GEM outer layer (A). Cross-section SEM micrographs of a control cylinder without drug (B) and cylinders containing $16 \mathrm{mg}$ OP (C) and $3 \mathrm{mg}$ GEM (D).

Abbreviations: GEM, gemcitabine; OP, oseltamivir phosphate; SEM, scanning electron microscopy. 


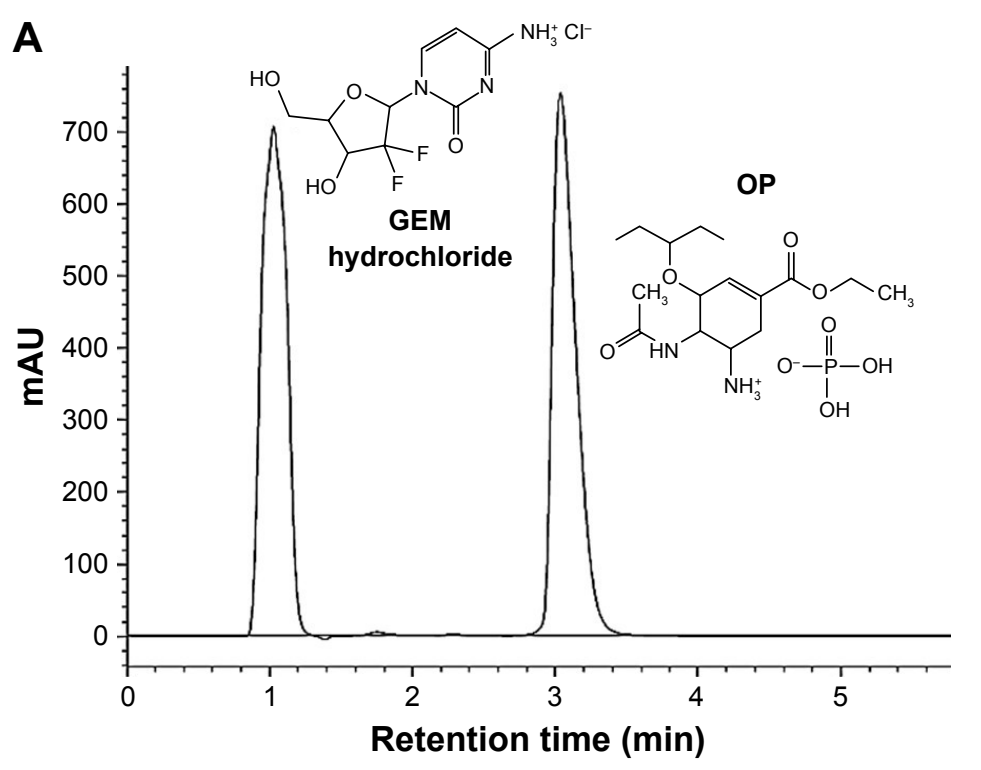

\section{B}

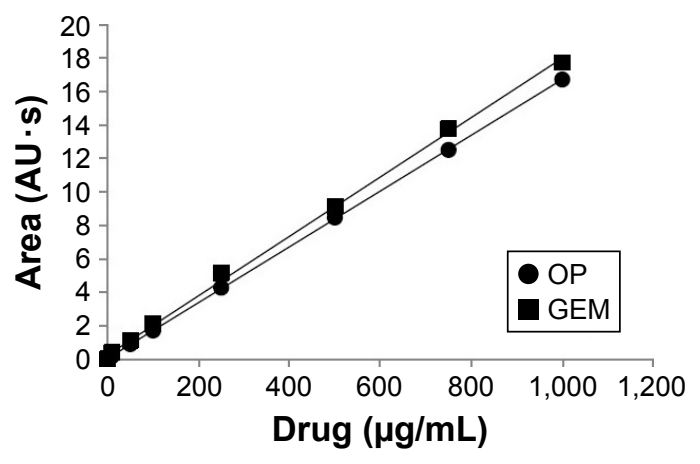

Figure 3 (A) OP and GEM were detected using a 1260 Infinity HPLC and a Poroshell 120 SB-CI 8 threaded column with mobile phase $60 \%$ methanol and $40 \% 0.04$ M ammonium acetate buffer ( $\mathrm{pH}$ 5.2). Retention times of GEM and OP were I.I and 3.0 min, respectively. (B) Calibration curve for OP and GEM using HPLC. Abbreviations: GEM, gemcitabine; HPLC, high performance liquid chromatograph; OP, oseltamivir phosphate.

linear reduction in the concentration of OP was observed concomitant with the emergence of a new peak at a retention time of $1.3 \mathrm{~min}$ with a linear profile (Figure 4). OP stability was also determined in $0.1 \%$ trifluoroacetic acid as stabilizer, showing a consistent stability over the same 30-day period. Clinically, OP used as an antiviral drug is administered orally as a prodrug, which is subsequently converted to oseltamivir carboxylate (OC) through ester bond hydrolysis by liver enzymes. ${ }^{12} \mathrm{OC}$ was analyzed using HPLC and found to have a retention time of $1.3 \mathrm{~min}$, matching that of the emerging peak when testing OP stability. The shorter retention time of OC compared to OP was expected, as OC is a more polar molecule. Thus, it is likely that the apparent decrease in OP over time is due to gradual hydrolysis to OC; yet, $70 \%$ of the OP remains detectable, even after 30 days at $37^{\circ} \mathrm{C}$. While the OC form is effective as an antiviral, the OP form has been shown most effective when directed against tumor tissues. ${ }^{7}$

It is noteworthy that OP- and GEM-loaded cylinders following incubation in cell culture medium for 15 days showed full stability of retained OP and GEM (Figure 4). Thus, PLGA encapsulation shows a dramatic stabilizing effect on the particulate form of OP for extended term prior to release.

\section{Release of OP and GEM from double- layered PLGA cylinders}

Several formulations were developed to optimize OP and GEM release from PLGA cylinders and are summarized in Table 1. The goal in designing the cylinders was to achieve a release profile that paralleled preclinical animal in vivo experiments involving injectable OP in combination with GEM (unpublished data, Szewczuk's lab, August 2016). A frequent issue was a high release of OP, and to a lesser extent GEM, from the cylinders within the first 3 days. Films were initially held at $4^{\circ} \mathrm{C}$ overnight prior to rolling, which were found sticky suggesting residual acetone present in the films. To this end, residual acetone may diffuse out of the cylinder in aqueous buffer, leaving pores and channels to allow for the permeation of water, resulting in premature diffusion and release of the drugs from the cylinder. OP may be affected more than GEM because OP particles were found to be smaller in size as seen in Figure 2. To test this hypothesis, cylinders were made from films, which had been kept at $20^{\circ} \mathrm{C}$ for $72 \mathrm{~h}$. The resulting films were no longer sticky and more rigid than those kept at $4^{\circ} \mathrm{C}$ for overnight. Two double-layered PLGA cylinders showed sustained release over 30 days and were further studied and selected for in vitro cell testing.

The selected cylinders based on the results from Table 1 were double-layered with one drug contained in the inner layer, and the other drug in the outer layer, made from films kept for $72 \mathrm{~h}$ at room temperature prior to rolling. Individual layers were determined to contain either $16 \mathrm{mg}$ of OP or $3 \mathrm{mg}$ of GEM. Cylinders were identified as inner/ outer $\mathrm{OP}^{\text {in }} / \mathrm{GEM}^{\text {out }}$ or GEM ${ }^{\text {in }} / \mathrm{OP}^{\text {out }}$. All double-layered PLGA cylinders were fabricated with $80 \mathrm{mg}$ of PLGA and 4-6 mg of Span 80 for each layer. After 30 days in media, PLGA cylinders were found to be completely degraded.

The simultaneous release of OP and GEM from $\mathrm{OP}^{\text {in }} / \mathrm{GEM}^{\text {out }}$ or $\mathrm{GEM}^{\text {in }} / \mathrm{OP}^{\text {out }}$ cylinders is shown in Figure 5. 

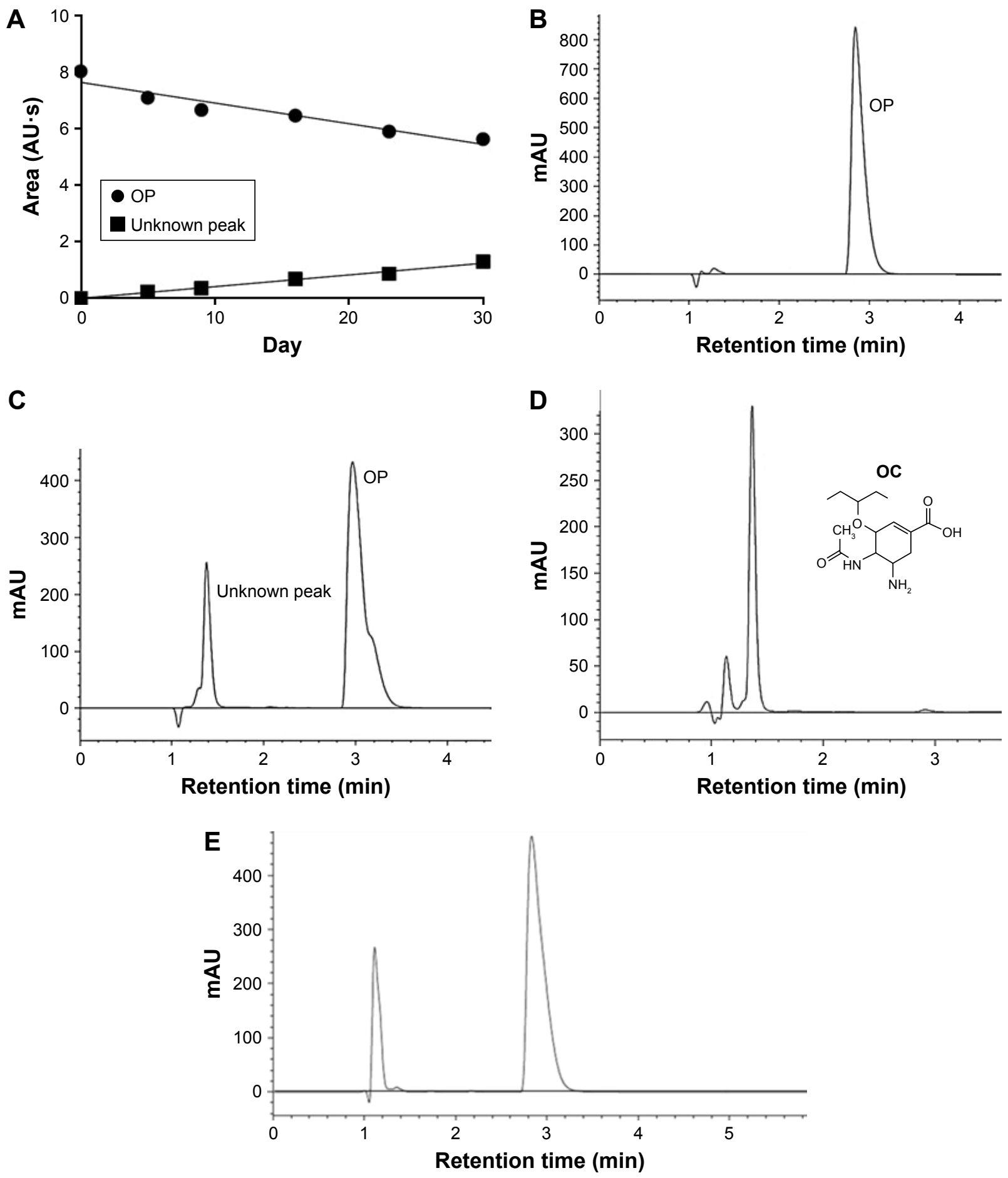

Figure 4 Area under curve of peak representing OP in $0.1 \mathrm{M}$ sodium phosphate buffer $(\mathrm{pH} 7.4)$ at $37^{\circ} \mathrm{C}$ (A). HPLC chromatograms of OP in sodium phosphate buffer at $37^{\circ} \mathrm{C}$ on day 0 (B) and day 30 (C). HPLC chromatogram of OC (D). HPLC chromatogram of GEM (left peak) and OP (right peak) still encapsulated in an OPin/GEMout cylinder following 15 days in PANCI cell culture (E).

Abbreviations: GEM, gemcitabine; OC, oseltamivir carboxylate; OP, oseltamivir phosphate.

$\mathrm{OP}^{\text {in }} / \mathrm{GEM}^{\text {out }}$ cylinders resulted in a higher initial release of GEM than OP with $39 \%$ of GEM and $15.5 \%$ of OP released during the initial $24 \mathrm{~h}$. GEM was then released at a constant rate until day 25 at which point $99 \%$ GEM was released from the cylinder. In addition, most of the OP was released between days 3 and 16 before beginning to plateau as seen in Figure 5A.

$\mathrm{GEM}^{\text {in }} / \mathrm{OP}^{\text {out }}$ cylinders showed a slower release of GEM as shown in Figure 5B. After $72 \mathrm{~h}$, only 2.5\% of GEM had been released from the cylinder. GEM release was linear 
Table I Formulations of PLGA cylinders containing OP and GEM and drug release from cylinders after $72 \mathrm{~h}$ in $0.1 \mathrm{M}$ phosphate buffer $(\mathrm{pH} 7.4)$ at $37^{\circ} \mathrm{C}$

\begin{tabular}{|c|c|c|c|c|c|c|}
\hline $\begin{array}{l}\text { Number } \\
\text { of layers }\end{array}$ & PLGA (mg) & $\begin{array}{l}\text { Layer arrangement, } \\
\text { inner/outer }\end{array}$ & OP (mg) & GEM (mg) & $\begin{array}{l}\text { Film } \\
\text { storage }\end{array}$ & $\begin{array}{l}\text { Release after } \\
72 \mathrm{~h}(\%)\end{array}$ \\
\hline \multirow[t]{6}{*}{ I } & 80 & - & 16 & 3 & $24 \mathrm{~h}, 4^{\circ} \mathrm{C}$ & OP: 86 \\
\hline & & & & & & GEM: $6 \mathrm{I}$ \\
\hline & 160 & - & 16 & 3 & $24 \mathrm{~h}, 4^{\circ} \mathrm{C}$ & OP: 86 \\
\hline & & & & & & GEM: 72 \\
\hline & 320 & - & 16 & 3 & $24 \mathrm{~h}, 4^{\circ} \mathrm{C}$ & OP: 58 \\
\hline & & & & & & GEM: 31 \\
\hline \multirow[t]{4}{*}{2} & 160 (80 mg/layer) & OP/GEM & 8 & 1.5 & $24 \mathrm{~h}, 4^{\circ} \mathrm{C}$ & OP: 3 \\
\hline & & & & & & GEM: 36 \\
\hline & & GEM/OP & 8 & 1.5 & $24 \mathrm{~h}, 4^{\circ} \mathrm{C}$ & OP: 12 \\
\hline & & & & & & GEM: I \\
\hline \multirow[t]{4}{*}{2} & 160 (80 mg/layer) & OP/GEM & 16 & 3 & $24 \mathrm{~h}, 4^{\circ} \mathrm{C}$ & OP: 87 \\
\hline & & & & & & GEM: 18 \\
\hline & & GEM/OP & 16 & 3 & $24 \mathrm{~h}, 4^{\circ} \mathrm{C}$ & OP: 73 \\
\hline & & & & & & GEM: 3 \\
\hline \multirow[t]{4}{*}{2} & 160 (80 mg/layer) & OP/GEM & 16 & 3 & $72 \mathrm{~h}, 20^{\circ} \mathrm{C}$ & OP: 21 \\
\hline & & & & & & GEM: 43 \\
\hline & & GEM/OP & 16 & 3 & $72 \mathrm{~h}, 20^{\circ} \mathrm{C}$ & OP: 53 \\
\hline & & & & & & GEM: 2 \\
\hline
\end{tabular}

Notes: Double-layered PLGA cylinders in which the OP was contained within the inner cylinder and the GEM was contained within the outer cylinder are designated as OP/ GEM, and cylinders in which the GEM was contained within the inner cylinder and the OP was contained within the outer cylinder are designated as GEM/OP. '-' indicates not applicable as there was only one inner/outer layer arrangement.

Abbreviations: GEM, gemcitabine; OP, oseltamivir phosphate; PLGA, poly(D,L-lactic-co-glycolic acid).

from day 3 until day 25 , when it slowed until day 30 . OP was released more quickly, with 13,34 , and $53 \%$ released from the cylinder after 24,48 , and $72 \mathrm{~h}$, respectively. OP release was then linear until day 20 , after which $97 \%$ of OP was released with the remaining amount released over the final 10 days.

The release of OP and GEM from double-layered PLGA cylinders was compared with the projected theoretical release of OP and GEM from a cylinder containing $16 \mathrm{mg}$ of OP and $3 \mathrm{mg}$ of GEM, assuming a linear rate of release over 30 days, as shown in Figure 6. Since the projected release was based on injectable drug treatment dosages that resulted in the stagnation or reduction of tumor volume in a heterotropic xenograft model of human pancreatic cancer in mice (unpublished data, Szewczuk's lab, August 2016), cylinders with the release of OP and GEM that closely matched the projected release were thought most likely to be effective. Indeed, the release profile of GEM from both
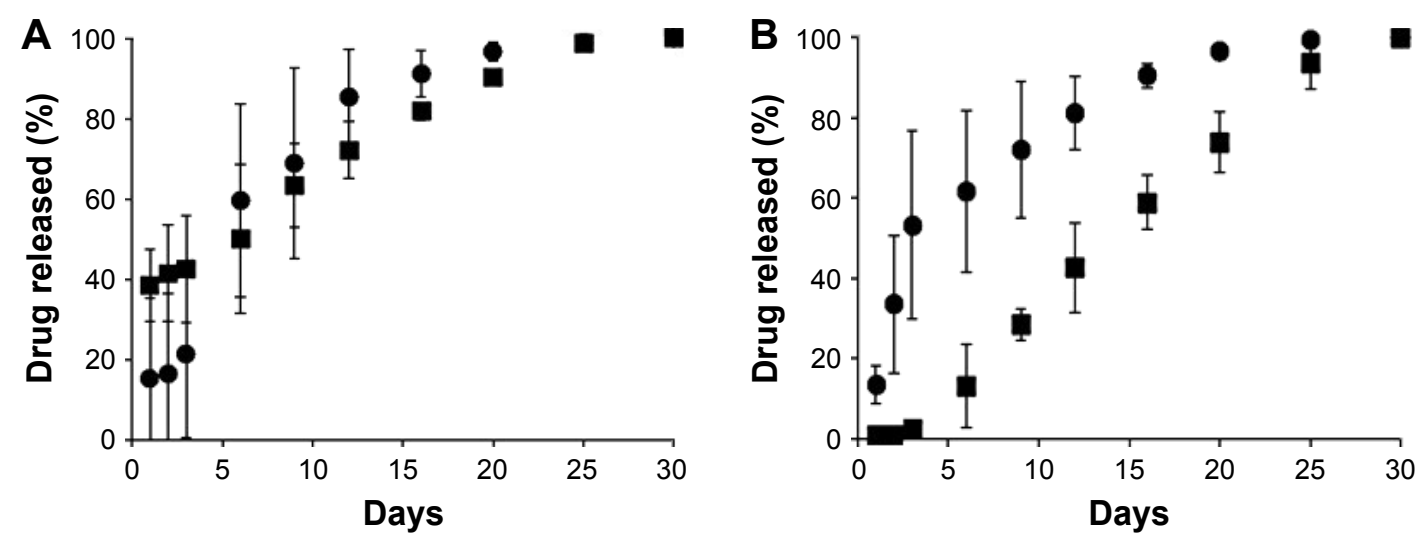

OP $\square$ GEM

Figure 5 Simultaneous release of OP and GEM from inner/outer for OPin/GEM out (A) and GEMin/OPout (B) double-layered PLGA cylinders.

Notes: Films were held at $20^{\circ} \mathrm{C}$ for $72 \mathrm{~h}$ prior to cylinder rolling. Data points represent the mean \pm standard deviation, $\mathrm{n}=3$ separate experiments. OP and GEM released are reported as percentage of the total amount of drug released after 30 days.

Abbreviations: GEM, gemcitabine; OP, oseltamivir phosphate. 

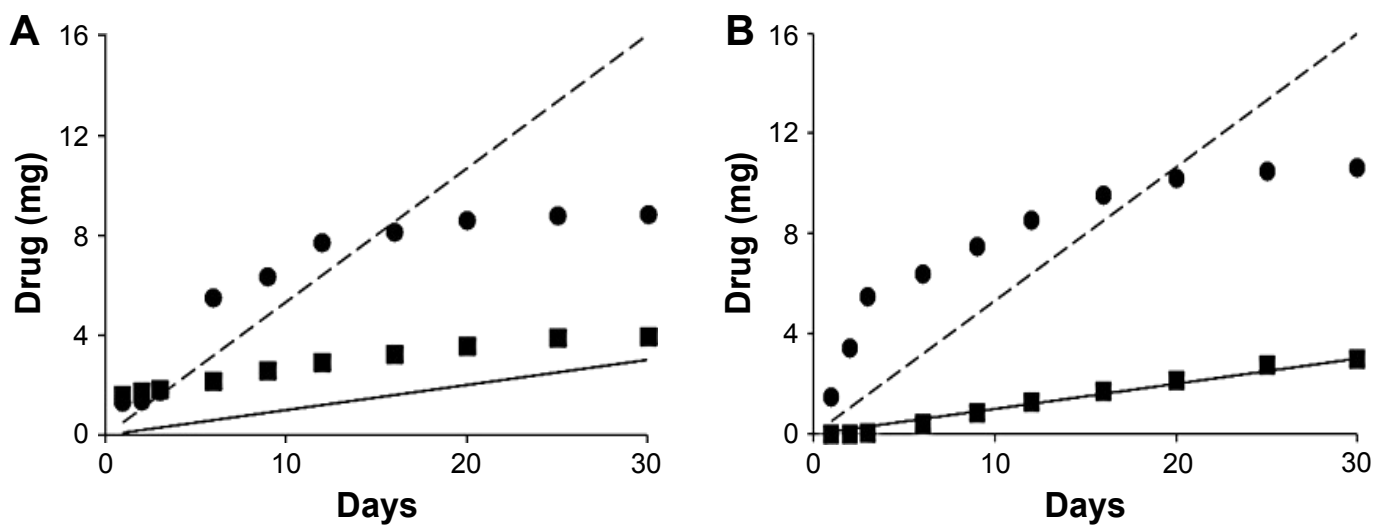

Figure 6 Release profile of OP $(\bullet)$ and GEM ( $\bullet$ ) from inner/outer OPin/GEMout $(\mathbf{A})$ and GEM ${ }^{\text {in/OPout }}(\mathbf{B})$ double-layered PLGA cylinders in comparison to the projected theoretical release of OP (- - -) and GEM (-), which assumes a constant release rate over 30 days.

Notes: The projected theoretical release rate was calculated from injected treatment doses of the two drugs shown effective in either the stagnation or reduction of tumor volume in a heterotopic xenograft model of human pancreatic cancer in mice (unpublished data, Szewczuk's lab, August 2016).

Abbreviations: GEM, gemcitabine; OP, oseltamivir phosphate.

types of cylinders is closely matched to the projected GEM release. The release of OP from both cylinders is similar to the projected release for the first 16-20 days, before the rate of release decreases and plateaus. Overall, both cylinders are similar to the projected release profile and selected for cell viability assays.

\section{Viability of PANCI and GEM-resistant PANCI cells exposed to OP and GEM released from PLGA cylinders}

The potential antitumor effects of OP and GEM released from $\mathrm{OP}^{\mathrm{in}} / \mathrm{GEM}^{\text {out }}$ or $\mathrm{GEM}^{\text {in }} / \mathrm{OP}^{\text {out }}$ cylinders on the viability of human pancreatic PANC1 carcinoma cells and their GEM resistant variants (PANC1-GEMR) were studied by monitoring numbers of viable cells, following exposure. Cell viability assays were initially performed using WST-1 reagent; however, interactions with the acidic polymer degradation products (glycolic acid and lactic acid) led to aberrant results. Instead, culture medium containing empty cylinder, OP ${ }^{\text {in }} / \mathrm{GEM}^{\text {out }}$ cylinder, or $\mathrm{GEM}^{\text {in }} / \mathrm{OP}^{\text {out }}$ cylinder was changed every 3 days, and trypan blue was used to count viable stained cells. Viable cells are shown as percent of the control empty PLGA cylinder group.

Images of PANC1 cells over 15 days culture depicted in Figure 7A show cells exposed to drug released from OPin/ $\mathrm{GEM}^{\text {out }}$ or $\mathrm{GEM}^{\text {in/OP }}{ }^{\text {out }}$ cylinders began to display an altered morphology from day 6 onward. Cells became elongated with an increase in spindle-like projections. When exposed to an empty blank cylinder, they appeared to have the same morphological feature as the untreated cells at each indicated time points, suggesting that degradable PLGA polymer alone had no direct effect on PANC1 cell morphology.

Cells treated with GEM $^{\text {in }} / \mathrm{OP}^{\text {out }}$ cylinder showed the lowest viable cell numbers at 3 and 6 days with 15.2 and $3.9 \%$, respectively, compared with untreated cells as seen in Figure 7B. For OPin/GEM ${ }^{\text {out }}$ exposed cells, the viable cell numbers on days 3 and 6 were 49.0 and $21.5 \%$, respectively, compared to that of untreated cells and, thus, GEM ${ }^{\text {in }} / \mathrm{OP}^{\text {out }}$ cylinders appeared to be more effective in preventing the cell growth of PANC1 over 3-6 days. However, PANC1 cells exposed to $\mathrm{OP}^{\mathrm{in}} / \mathrm{GEM}^{\text {out }}$ cylinders had lower viable cell counts after 10 and 15 days, showing 10.8 and $1.2 \%$ of viable cells, respectively. When exposed to OP $/ \mathrm{GEM}^{\text {out }}$ cylinders, they remained viable compared to the untreated cells, while 11.7 and $12.2 \%$ of cells exposed to $\mathrm{GEM}^{\mathrm{in}} / \mathrm{OP}^{\text {out }}$ cylinders remained viable.

High cell viability and no visible changes in cell morphology were observed in PANC1 exposed to blank cylinders compared to the untreated cells. PLGA alone does not appear to have an effect on PANC1 cells, and thus, any changes in morphology or cell viability following treatment with cylinders are due to the effect of released OP and GEM.

In the case of GEM-resistant cells (PANC1-GEMR) exposed to $\mathrm{OP}^{\mathrm{in}} / \mathrm{GEM}^{\text {out }}$ or $\mathrm{GEM}^{\text {in }} / \mathrm{OP}^{\text {out }}$ cylinders, little cell growth appeared to occur after 3 days as seen in Figure 8A. Thus, few cells survived following exposure $>3$ days, as seen in Figure 8B. PANC1-GEMR cells exposed to blank cylinders showed a similar morphology to untreated cells, indicating again that the PLGA did not affect the viability of the drug-resistant variant cells. 

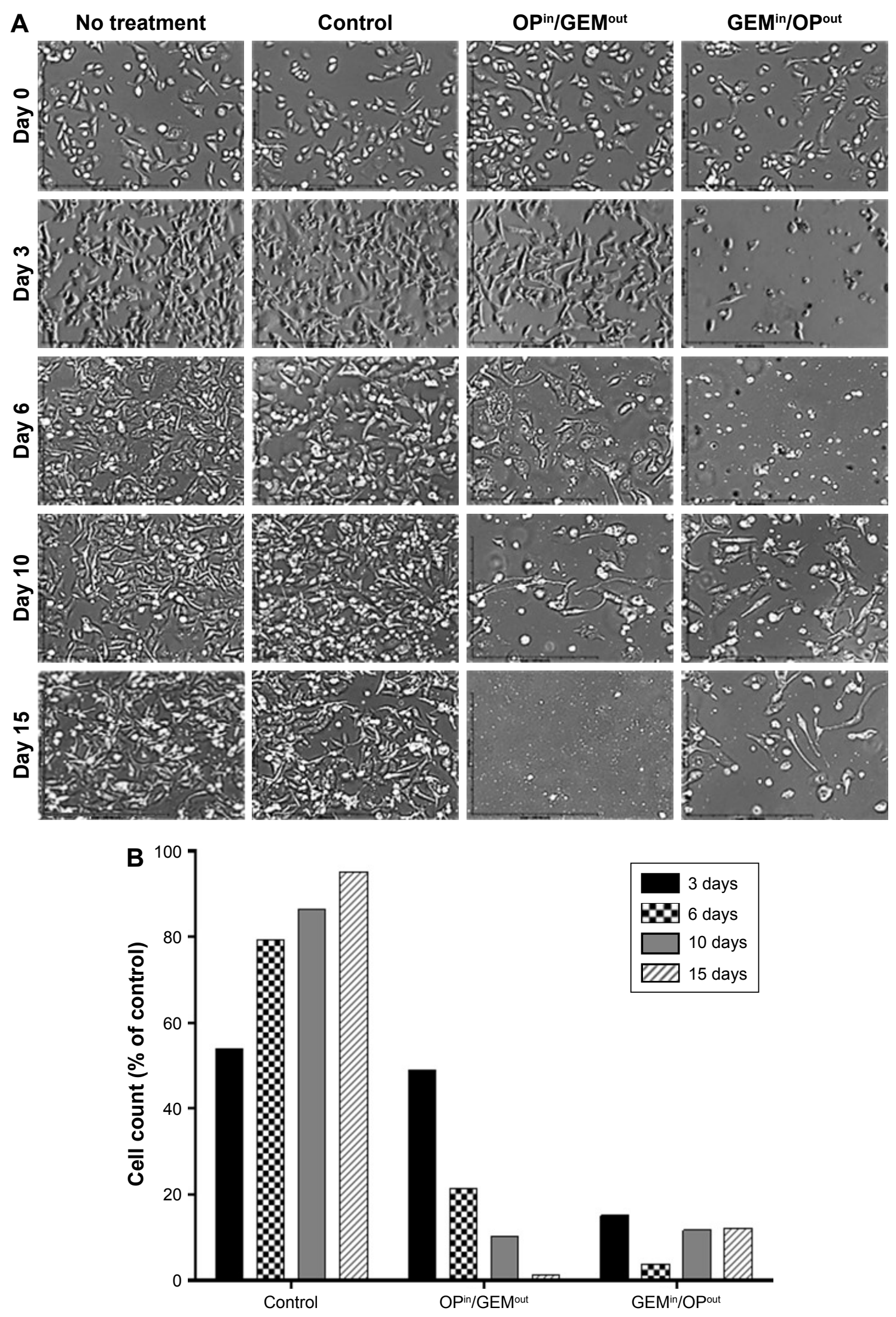

Figure 7 (A) PANCI untreated cells over 15 days, compared to PANCI exposed to drug-free blank, and inner/outer OPin/GEM ${ }^{\text {out, }}$, or GEMin/OPout cylinders. Scale bar: $508 \mu$ m. (B) Viable cell numbers are expressed as a percentage of the untreated cells. The data are a representation of one out of two independent experiments showing similar results. Abbreviations: GEM, gemcitabine; OP, oseltamivir phosphate. 

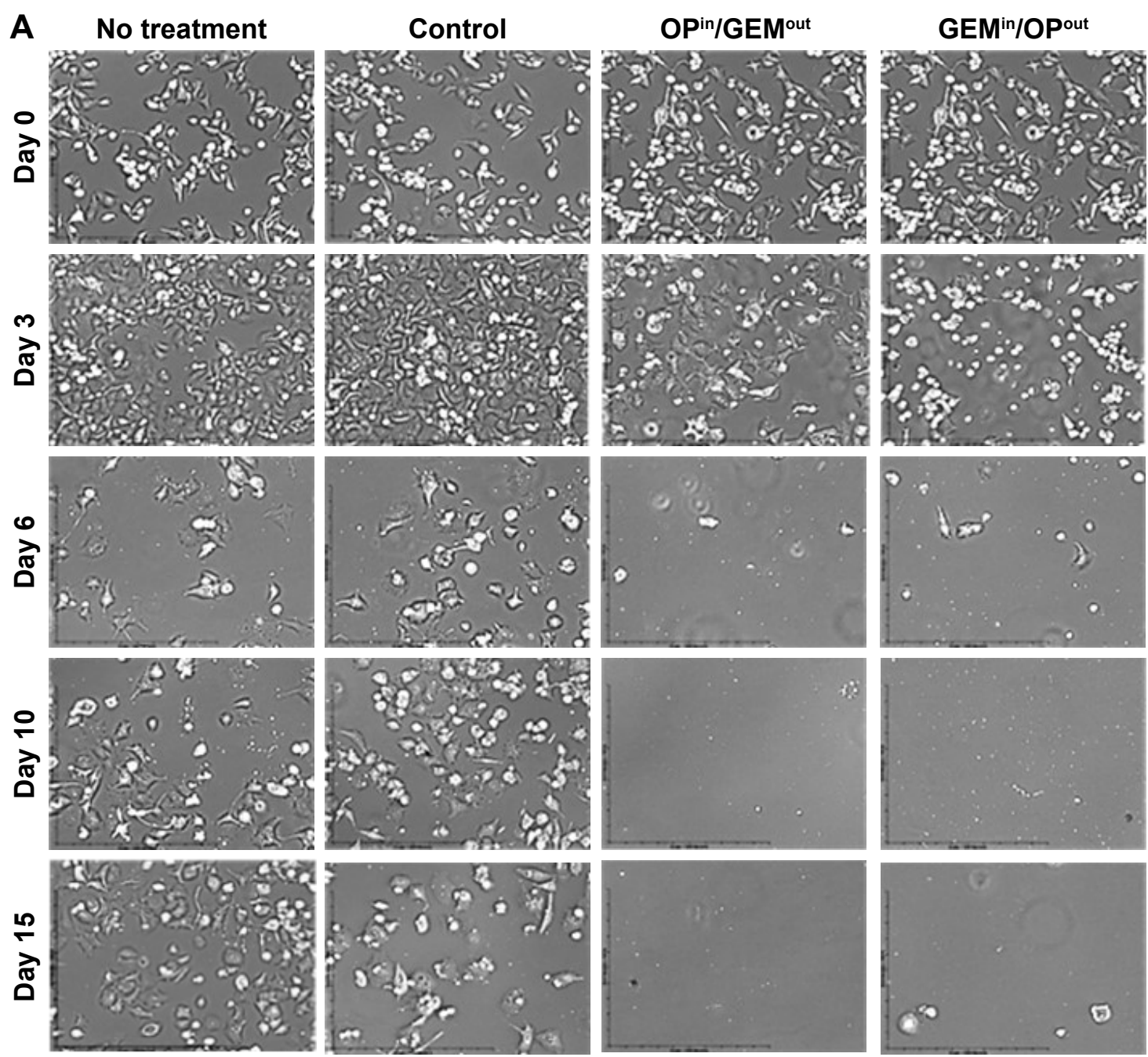

B

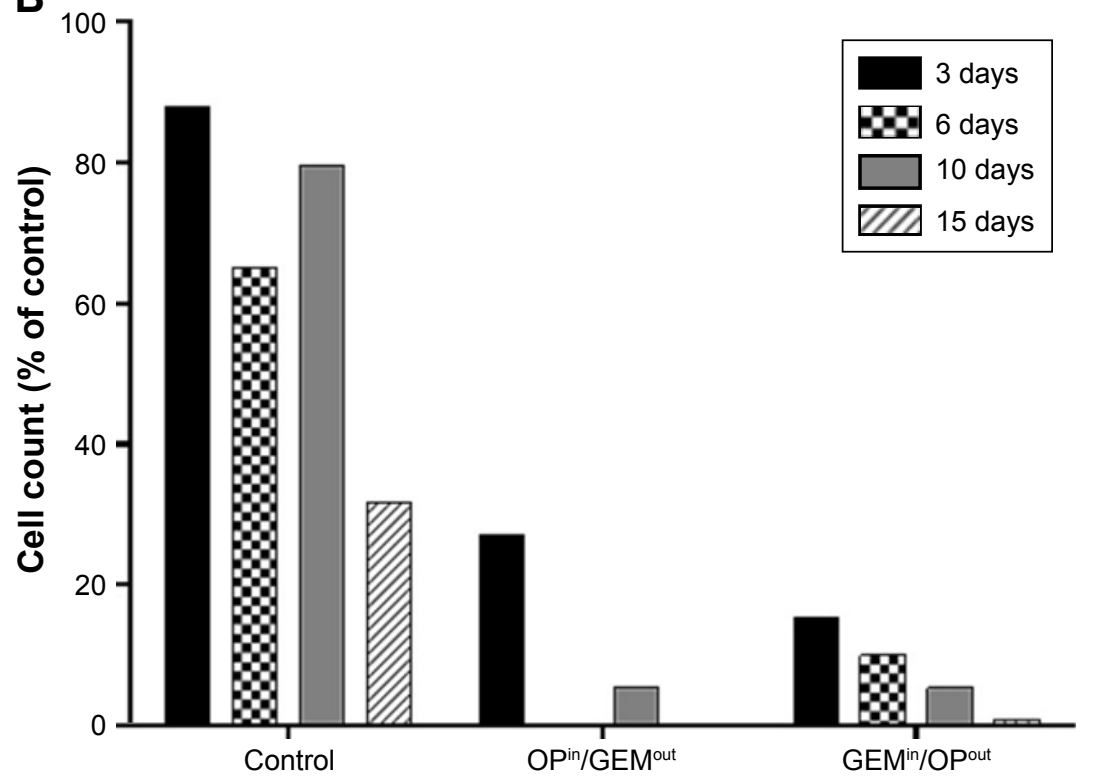

Figure 8 PANCI-GEMR untreated cells over I5 days compared to PANCI-GEMR cells exposed to drug-free blank and inner/outer OPin/GEMout or GEMin/OPout cylinders (A). Scale bar: $508 \mu \mathrm{m}$. Viable cell numbers are expressed as percentage of the untreated cells (B). Absence of a bar indicates that no viable cells were present. The data are the representation of one out of two independent experiments showing similar results.

Abbreviations: GEM, gemcitabine; OP, oseltamivir phosphate. 
$\mathrm{OP}$ and GEM released from $\mathrm{OP}^{\text {in }} / \mathrm{GEM}^{\text {out }}$ and $\mathrm{GEM}^{\text {in }} / \mathrm{OP}^{\text {out }}$ cylinders greatly reduced the number of PANC1-GEMR viable cells at all time points. For PANC1 cells, viability was lower for cells treated with the $\mathrm{GEM}^{\text {in }} / \mathrm{OP}^{\text {out }}$ cylinders compared to the $\mathrm{OP}$ in/GEM ${ }^{\text {out }}$ cylinders after 3 days of exposure. However, treatment with $\mathrm{OP}^{\text {in }} / \mathrm{GEM}^{\text {out }}$ cylinders resulted in equal or lower cell counts compared to $\mathrm{GEM}^{\text {in }} / \mathrm{OP}^{\text {out }}$ cylinders at all other time points.

Release of OP and GEM from the OP ${ }^{\text {in }} / \mathrm{GEM}^{\text {out }}$ cylinders appeared to be most effective at reducing cell viability for both PANC1 and PANC1-GEMR cells over the 15 days period. The experiments described earlier were repeated with the OPin/GEM ${ }^{\text {out }}$ cylinders on both cell types, showing reproducible similar results (data not shown). The OPin GEM $^{\text {out }}$ cylinders dramatically reduced the number of viable cells for both cell types and at each time point.

In addition to demonstrating reduction in PANC1 and PANC1-GEMR target cell viability during exposure to the combinatorial and sequential release OP and GEM, it is instructive to consider the potential for concomitant migratory or metastatic phenotype. Previously, O'Shea et $\mathrm{al}^{8}$ treated PANC1 and PANC1-GEMR with $600 \mu \mathrm{g} / \mathrm{mL}$ OP for $24 \mathrm{~h}$ and observed a significant increase in E-cadherin expression as well as a significant decrease in $\mathrm{N}$-cadherin expression, consistent with epithelial-mesenchymal transition (EMT), indicating a more metastatic phenotype than untreated PANC1 cells. ${ }^{13,14}$ In the present study, differences in the response to OP and GEM released from the $\mathrm{OP}$ in $/ \mathrm{GEM}^{\text {out }}$ cylinders compared to the $\mathrm{GEM}^{\text {in/OP }} \mathrm{OP}^{\text {out }}$ cylinders may be due to the different cylinder release profiles as shown in Figure 5. The $\mathrm{GEM}^{\text {in }} / \mathrm{OP}^{\text {out }}$ cylinders have a higher initial release of OP and a lower initial release of GEM than the $\mathrm{OP}^{\mathrm{in}} / \mathrm{GEM}^{\text {out }}$ cylinders. Therefore, it is proposed that the higher initial release of OP from $\mathrm{GEM}^{\text {in }} / \mathrm{OP}^{\text {out }}$ cylinders could also induce a reversal of EMT.

Chemotherapeutic treatment of pancreatic cancer is often hindered by chemoresistance, which is often linked to EMT in many cancers, ${ }^{13,15}$ including pancreatic cancer. ${ }^{16-18}$ EMT is characterized by reduced cell-cell adhesion, reorganization of the cytoskeleton, and loss of cell polarity, resulting in cells that have a more migratory phenotype ${ }^{19}$ Cells undergoing EMT have decreased expression of epithelial markers such as E-cadherin, occludin, cytokeratins, and desmoplakin, while showing an increased expression of the mesenchymal markers $\mathrm{N}$-cadherin, vimentin, and fibronectin. ${ }^{13,20-22}$ Transcription factors, such as Slug, Snail, Twist, and Zeb-1, are repressors of E-cadherin, and their upregulation has also been linked to chemoresistance and EMT..$^{15,17,18,23}$ We have previously demonstrated that $\mathrm{OP}$ can restore chemosensitivity and induce mesenchymal to epithelial transition. ${ }^{8}$ In the present work, we have observed that the images of the surviving cells following $\mathrm{OP}^{\text {in }} / \mathrm{GEM}^{\text {out }}$ exposure from PLGA cylinders showed altered morphologies that could be consistent with sustained chemosensitivity and induced mesenchymal to epithelial transition.

\section{Conclusion}

The double-layered PLGA cylinders developed in the present study provide the proof of concept of long-term delivery of OP and GEM from a biodegradable, implantable device for the possible treatment of cancer. Double-layered PLGA cylinders containing OP and GEM showed not only a sustained release of both drugs over 30 days but also stability of the drugs. OP and GEM released from OP $/ \mathrm{GEM}^{\text {out }}$ and $\mathrm{GEM}^{\text {in }} / \mathrm{OP}^{\text {out }}$ cylinders strongly reduced PANC1 and PANC1GEMR cell viability over a period of 3-15 days, with some evidence supporting previous observations of sustained chemosensitivity and metastatic phenotype of PANC1 cells. The double-layered PLGA cylinders are an effective delivery system for the sustained release of OP and GEM due to a simple fabrication process, the high loading of the small molecular weight hydrophilic drugs, the stability of the drugs while encapsulated, and the potential for implantation and localized drug delivery.

\section{Acknowledgments}

This study was supported in part by grants to MRS and RJN from the Natural Sciences and Engineering Research Council of Canada and private sector cancer funding from the Josefowitz Family to MRS. SAL was the recipient of the Queen's Graduate Award (QGA). Present address of SAL: Polymer Science Group, Department of Chemical and Biomolecular Engineering, University of Melbourne, Parkville, Victoria 3010, Australia.

\section{Author contributions}

SAL and AJB fabricated and characterized the doublelayered PLGA cylinders. SAL generated and maintained cancer cell line, confirmed the release studies of OP and GEM, and carried out in vitro study with the cells in the presence of PLGA. MRS and RJN supervised the research design and prepared the manuscript with SAL. All authors contributed toward data analysis, drafting, and revising the article and agree to be accountable for all aspects of the work.

\section{Disclosure}

The authors report no conflicts of interest in this work. 


\section{References}

1. Obara K, Ishihara M, Ozeki Y, et al. Controlled release of paclitaxel from photocrosslinked chitosan hydrogels and its subsequent effect on subcutaneous tumor growth in mice. J Control Release. 2005; 110(1):79-89.

2. Yapp DT, Wong MQ, Kyle AH, et al. The differential effects of metronomic gemcitabine and antiangiogenic treatment in patient-derived xenografts of pancreatic cancer: treatment effects on metabolism, vascular function, cell proliferation, and tumor growth. Angiogenesis. 2016;19(2):229-244.

3. Laquente B, Lacasa C, Ginesta MM, et al. Antiangiogenic effect of gemcitabine following metronomic administration in a pancreas cancer model. Mol Cancer Ther. 2008;7(3):638-647.

4. Cham KK, Baker JH, Takhar KS, et al. Metronomic gemcitabine suppresses tumour growth, improves perfusion, and reduces hypoxia in human pancreatic ductal adenocarcinoma. Br J Cancer. 2010;103(1): 52-60.

5. Almoguera C, Shibata D, Forrester K, Martin J, Arnheim N, Perucho M. Most human carcinomas of the exocrine pancreas contain mutant c-Kras genes. Cell. 1988;53(4):549-554.

6. Burris H, Storniolo AM. Assessing clinical benefit in the treatment of pancreas cancer: gemcitabine compared to 5-fluorouracil. Eur J Cancer. 1997;33(supp1 1):S18-S22.

7. Amith SR, Jayanth P, Franchuk S, et al. Dependence of pathogen molecule-induced toll-like receptor activation and cell function on Neu1 sialidase. Glycoconj J. 2009;26(9):1197-1212.

8. O'Shea LK, Abdulkhalek S, Allison S, Neufeld RJ, Szewczuk MR. Therapeutic targeting of Neul sialidase with oseltamivir phosphate (Tamiflu(R)) disables cancer cell survival in human pancreatic cancer with acquired chemoresistance. Onco Targets Ther. 2014;7: 117-134.

9. Hrynyk M, Ellis JP, Haxho F, et al. Therapeutic designed poly (lacticco-glycolic acid) cylindrical oseltamivir phosphate-loaded implants impede tumor neovascularization, growth and metastasis in mouse model of human pancreatic carcinoma. Drug Des Devel Ther. 2015;9: 4573-4586.

10. Nair LS, Laurencin CT. Biodegradable polymers as biomaterials. Prog Polym Sci. 2007;32(8-9):762-798.
11. Danhier F, Ansorena E, Silva JM, Coco R, Le Breton A, Preat V. PLGA-based nanoparticles: an overview of biomedical applications. J Control Release. 2012;161(2):505-522.

12. Shi D, Yang J, Yang D, et al. Anti-influenza prodrug oseltamivir is activated by carboxylesterase human carboxylesterase 1 , and the activation is inhibited by antiplatelet agent clopidogrel. J Pharmacol Exp Ther. 2006;319(3):1477-1484.

13. Creighton CJ, Gibbons DL, Kurie JM. The role of epithelialmesenchymal transition programming in invasion and metastasis: a clinical perspective. Cancer Manag Res. 2013;5:187-195.

14. Thiery JP, Acloque H, Huang RY, Nieto MA. Epithelial-mesenchymal transitions in development and disease. Cell. 2009;139(5):871-890.

15. Martin TA, Goyal A, Watkins G, Jiang WG. Expression of the transcription factors snail, slug, and twist and their clinical significance in human breast cancer. Ann Surg Oncol. 2005;12(6):488-496.

16. Shah AN, Summy JM, Zhang J, Park SI, Parikh NU, Gallick GE. Development and characterization of gemcitabine-resistant pancreatic tumor cells. Ann Surg Oncol. 2007;14(12):3629-3637.

17. Arumugam T, Ramachandran V, Fournier KF, et al. Epithelial to mesenchymal transition contributes to drug resistance in pancreatic cancer. Cancer Res. 2009;69(14):5820-5828.

18. Hotz B, Arndt M, Dullat S, Bhargava S, Buhr HJ, Hotz HG. Epithelial to mesenchymal transition: expression of the regulators snail, slug, and twist in pancreatic cancer. Clin Cancer Res. 2007;13(16):4769-4776.

19. Hugo H, Ackland ML, Blick T, et al. Epithelial-mesenchymal and mesenchymal-epithelial transitions in carcinoma progression. $J$ Cell Physiol. 2007;213(2):374-383.

20. Weber CE, Li NY, Wai PY, Kuo PC. Epithelial-mesenchymal transition, TGF-beta, and osteopontin in wound healing and tissue remodeling after injury. J Burn Care Res. 2012;33(3):311-318.

21. Bhowmick NA, Ghiassi M, Bakin A, et al. Transforming growth factorbeta 1 mediates epithelial to mesenchymal transdifferentiation through a RhoA-dependent mechanism. Mol Biol Cell. 2001;12(1):27-36.

22. Nieman MT, Prudoff RS, Johnson KR, Wheelock MJ. N-cadherin promotes motility in human breast cancer cells regardless of their E-cadherin expression. J Cell Biol. 1999;147(3):631-644.

23. Cano A, Perez-Moreno MA, Rodrigo I, et al. The transcription factor snail controls epithelial-mesenchymal transitions by repressing E-cadherin expression. Nat Cell Biol. 2000;2(2):76-83.
Drug Design, Development and Therapy

\section{Publish your work in this journal}

Drug Design, Development and Therapy is an international, peerreviewed open-access journal that spans the spectrum of drug design and development through to clinical applications. Clinical outcomes, patient safety, and programs for the development and effective, safe, and sustained use of medicines are the features of the journal, which

\section{Dovepress}

has also been accepted for indexing on PubMed Central. The manuscript management system is completely online and includes a very quick and fair peer-review system, which is all easy to use. Visit http://www.dovepress.com/testimonials.php to read real quotes from published authors. 